\title{
NONDESTRUCTIVE FLUORESCENCE LIFETIME IMAGING AND TIME-RESOLVED FLUORESCENCE SPECTROSCOPY DETECT CARTILAGE MATRIX DEPLETION AND CORRELATE WITH MECHANICAL PROPERTIES
}

\author{
A.K. Haudenschild ${ }^{1}$, B.E. Sherlock ${ }^{1}$, X. Zhou ${ }^{1}$, J.C. Hu${ }^{2}$, J.K. Leach ${ }^{1,3}$, L. Marcu ${ }^{1}$ and K.A. Athanasiou ${ }^{2, *}$ \\ ${ }^{1}$ Department of Biomedical Engineering, University of California, Davis, CA, USA \\ ${ }^{2}$ Department of Biomedical Engineering, University of California, Irvine, CA, USA \\ ${ }^{3}$ Department of Orthopedic Surgery, University of California, Davis Medical Center, Sacramento, CA, USA
}

\begin{abstract}
Tissue engineers utilize a battery of expensive, time-consuming and destructive techniques to assess the composition and function of engineered tissues. A nondestructive solution to monitor tissue maturation would reduce costs and accelerate product development. As a first step toward this goal, two nondestructive, label-free optical techniques, namely multispectral fluorescent lifetime imaging (FLIm) and time-resolved fluorescence spectroscopy (TRFS), were investigated for their potential in evaluating the biochemical and mechanical properties of articular cartilage.

Enzymatic treatments were utilized to selectively deplete cartilage of either collagen or proteoglycan, to produce a range of matrix compositions. Samples were assessed for their optical properties using a fibercoupled optical system combining FLIm and TRFS, their biochemical and mechanical properties and by histological staining. Single and multivariable correlations were performed to evaluate relationships among these properties.

FLIm- and TRFS-derived measurements are sensitive to changes in cartilage matrix and correlate with mechanical and biochemical assays. Mean fluorescence lifetime values extracted from FLIm images (375$410 \mathrm{~nm}$ spectral band) showed strong, specific correlations with collagen content $\left(R^{2}=0.79, p<0.001\right)$ and tensile properties $\left(R^{2}=0.45, p=0.02\right)$. TRFS lifetime measurements centered at $520 \mathrm{~nm}$ (with a $5 \mathrm{~nm}$ bandwidth) possessed strong, specific correlations with proteoglycan content $\left(R^{2}=0.59, p=0.001\right)$ and compressive properties $\left(R^{2}=0.71, p<0.001\right)$.

Nondestructive optical assessment of articular cartilage, using a combination of FLIm- and TRFS-derived parameters, provided a quantitative method for determining tissue biochemical composition and mechanical function. These tools hold great potential for research, industrial and clinical settings.
\end{abstract}

Keywords: Cartilage, biomechanics, extracellular matrix, collagens, proteoglycans, imaging, cartilage repair and regeneration, tissue engineering, regenerative medicine.

*Address for correspondence: Kyriacos A. Athanasiou, PhD, Department of Biomedical Engineering, University of California Irvine, 3418 Engineering Hall, Irvine, CA 92697, USA.

Telephone number: +1 9498249196 Fax number: +19498241727 E-mail: athens@uci.edu

Copyright policy: This article is distributed in accordance with Creative Commons Attribution Licence (http://creativecommons.org/licenses/by-sa/4.0/).

\section{Introduction}

Tissue engineering of functional replacement tissues requires a strict set of design criteria involving both biochemical and mechanical properties. Currently, tissue engineers, in both research and clinical setting, rely predominantly on destructive and time-consuming techniques that require complete or partial sample loss. Destructive measurements provide valuable information concerning the tissue biochemical and mechanical properties but do not allow spatial or temporal monitoring during tissue development. Furthermore, destructive assays of engineered tissues during in vivo experiments require sacrificing the experimental animal, while nondestructive alternatives have the potential to reduce animal use. Clinically, once the replacement tissue has been implanted in the patient, clinicians are often unable to monitor the biochemical and mechanical changes occurring. There is a clear 
need for nondestructive methods to evaluate tissue-engineered constructs at all levels of product development (Morgan et al., 2014).

To address these issues, quantitative optical techniques based on endogenous tissue autofluorescence are described for nondestructive tissue imaging and characterization (Gorpas et al., 2015; Gorpas et al., 2016; Sun et al., 2012). Several endogenous biomolecules, including the extracellular matrix (ECM) protein collagen (Georgakoudi et al., 2002) and its crosslinks (Wagnieres et al., 1998), generate tissue autofluorescence. Changes in the fluorescence emission profiles reflect changes in the relative concentration and distribution of individual fluorophores within the tissue. Two optical techniques that are based on fluorescence lifetime (LT) contrast are single-point time-resolved fluorescence spectroscopy (TRFS) - which records the temporal dynamics of ECM autofluorescence at high spectral resolution - and fluorescence lifetime imaging (FLIm) - which maps the surface distribution of the endogenous fluorophores to generate images of the tissue biochemistry. TRFS and FLIm detect biochemical changes in various biological tissues, both in vitro and in vivo (Fite et al., 2011; Manning et al., 2013; Yankelevich et al., 2014). The combination of TRFS and FLIm in a single apparatus allows for the characterization of cartilage ECM identity, quantity and distribution.

In addition to monitoring changes in biochemical composition, it is also necessary to determine changes in mechanical properties to determine the functionality of both native and engineered replacement tissues. Articular cartilage is a loadbearing tissue and sufficient tensile and compressive properties are critical for the success of engineered replacement tissue (Huey et al., 2012; Makris et al., 2015; Responte et al., 2007). The cartilage ECM is comprised primarily of collagen type II, proteoglycans and water (Zhang et al., 2009) and cartilage mechanical properties are attributed to these ECM components (Setton et al., 1993).

To characterize both the initial materials and the tissue development and maturation that follows, it is essential to have quantitative, validated tools, optimized specifically for articular cartilage tissue engineering applications (Morgan et al., 2014). In the current study, by selectively degrading the individual components of native cartilage ECM, a model for examining the structure-function relationships of articular cartilage and the changes associated with matrix formation/degradation was developed to provide a testing environment for novel diagnostic techniques.

The bacterial enzyme chondroitinase $\mathrm{ABC}$ (cABC) effectively degrades all three forms of sulfated glycosaminoglycans (GAGs): chondroitin sulfate, dermatan sulfate and chondroitin-6-sulfate (Hamai et al., 1997). Similarly, bacterial type II collagenase (ColG) degrades native collagen but exhibits broader substrate specificity (Chojkier et al., 1980).
The overall goal of this work was to study whether nondestructive optical methods had the potential to replace the conventional destructive measurements of tissue properties to reduce costs and research time and to allow for time-lapse measurements tracking the maturation of individual samples. Ultimately, endoscopic in vivo evaluations of tissue-engineered implants using these nondestructive technologies is desired (Requejo-Isidro et al., 2004).

The current study aimed to deplete the predominant articular cartilage matrix components, collagen and GAG, to measure changes in mechanical, biochemical and optical properties and to form specific, quantitative relationships between destructive and nondestructive measurements. By creating an array of cartilage samples with varying biochemical and mechanical properties, the following questions were addressed: which optical wavelengths were best suited for collagen and proteoglycan quantification, which optical parameters correlated with mechanical properties and wheather the strength of these correlations was sufficient to replace destructive testing methods.

The working hypothesis was that the label-free, nondestructive FLIm and TRFS optical techniques could be used to infer the changes in both biochemical composition and biomechanical properties of articular cartilage over a wide range of matrix compositions.

\section{Materials and Methods}

\section{Cartilage harvest}

Full thickness cartilage explants ( $8 \mathrm{~mm}$ in diameter) were harvested from the femoral condyles of four juvenile bovine stifle joints ( $n=19 /$ joint) (Research 87, Boston, MA, USA) (Fig. 1). Samples were trimmed to $2 \mathrm{~mm}$ thickness with a custom cutting jig, while preserving the articular surface, and wrapped in gauze soaked in phosphate buffered saline (PBS) (Sigma-Aldrich) with protease inhibitors, as previously described (Agemura et al., 1990). Samples were cycled through three freeze-thaw cycles to lyse cells and stored at $-20^{\circ} \mathrm{C}$ until treatment and testing.

For comparison of viable and frozen tissue, freshly harvested cylindrical samples $(n=6)$ were cut in crosssection and one half was imaged immediately (viable) while the second half underwent three freeze-thaw cycles (frozen) prior to optical assessment.

\section{Proteolytic enzyme treatments}

Cartilage explants were randomly assigned to a treatment group (Griffin et al., 2014; Karchner et al., 2018; Nissi Mj et al., 2016) and placed one sample per well in a 6-well plate (Costar, Corning) in $6 \mathrm{~mL}$ of PBS. To remove collagen, type II collagenase (ColG) (Worthington, Lakewood, NJ, USA) was added to a final concentration of 0 (control), 20 (low ColG) or $80 \mu \mathrm{g} / \mathrm{mL}$ (high ColG) per well. Similarly, to remove proteoglycans, chondroitinase $\mathrm{ABC}(\mathrm{cABC})$ (Sigma-Aldrich) was added to a final concentration 


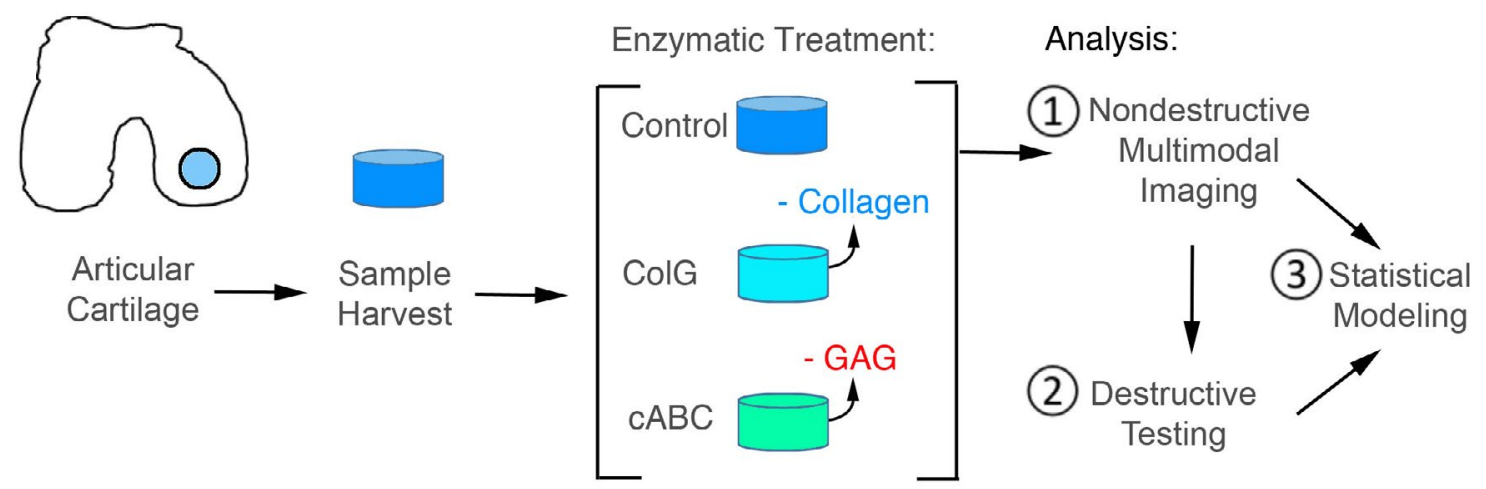

Fig. 1. Schematic diagram of the study. Harvest and treatment of bovine articular cartilage samples in untreated (control), ColG- or cABC-treated groups. Analysis performed on each sample included: 1) nondestructive imaging and spectroscopy (FLIm and TRFS), 2) destructive mechanical (compressive and tensile), biochemical (total collagen and proteoglycan) testing and 3) statistical modeling of measurement correlations to determine significant factors.

of 0 (control), 0.5 (low cABC) or $1 \mathrm{U} / \mathrm{mL}$ (high cABC) per well. All treatments included $50 \mathrm{U} / \mathrm{mL}$ saltactivated nuclease (ArcticZymes, Tromsø, Norway), $0.02 \%$ ethylenediaminetetraacetic acid (EDTA) (Life Technologies) and $1 \%$ penicillin/streptomycin/ fungizone (Lonza). Sample were treated for $10 \mathrm{~h}$ at $37^{\circ} \mathrm{C}$ on a rotatory plate at 35 revolutions $/ \mathrm{min}$. After treatment, samples were washed in fresh PBS for $12 \mathrm{~h}$ to remove residual enzymes.

To determine the applicability of optical assessment on viable tissue, freshly harvested constructs were processed in PBS (control), $80 \mu \mathrm{g} / \mathrm{mL}$ of collagenase (high $\mathrm{ColG}$ ) or $1 \mathrm{U} / \mathrm{mL}$ of $\mathrm{cABC}$ (high cABC) as described above.

\section{Non-destructive optical assessment of cartilage}

A fiber-based, fluorescence lifetime imaging and spectroscopy system was used for nondestructive assessment of the articular cartilage samples ( $n=6 /$ condition), followed by biochemical assays, mechanical evaluation and histological processing. The functional principle of operation of the FLIm and TRFS systems is reported by Sun et al. (2009) and Yankelevich et al. (2014). Time-resolved fluorescence was acquired by both systems using the pulse sampling technique (Marcu et al., 2014) and the decays were parameterized in post-processing using a constrained least-squares deconvolution with Laguerre expansion (Liu et al., 2012). Average lifetimes were calculated using the definition of intensity-weighted average lifetime (Yankelevich et al., 2014). FLIm and TRFS used the same microchip laser (STV-02E-1x0, TEEM photonics, Grenoble, France) that emitted optical pulses at a wavelength of $355 \mathrm{~nm}$ to generate sample autofluorescence. Light was delivered to and collected from the sample using a $2 \mathrm{~m}$ long, flexible fiber-optic cable (Molex, Lisle, IL, USA) with an outer diameter of $480 \mu \mathrm{m}$. During imaging, the distal tip of the fiber was positioned approximately $1 \mathrm{~mm}$ above and perpendicular to the articular surface. A two-axis digital translation stage (MX80L, Parker, Cleveland, OH, USA) was used to scan the fiber across the surface. For optical assessment, the central $5 \mathrm{~mm}$ diameter core from each sample was placed in a custom-made, glass sample holder in a PBS bath at room temperature.

\section{FLIm system}

For FLIm, the fluorescence emission was separated into four spectral bands $(\mathrm{CH} 1=375-410 \mathrm{~nm}$, $\mathrm{CH} 2=450-485 \mathrm{~nm}, \mathrm{CH} 3=515-565 \mathrm{~nm}, \mathrm{CH} 4=595-$ $660 \mathrm{~nm}$ ) using a custom-built wavelength selection module. Each spectral band had an associated fiber optic delay line of different lengths (longer wavelength bands have longer delay lines). This arrangement allowed the four spectral bands to be temporally multiplexed onto a single photomultiplier tube (PMT) (R5916U-50, Hamamatsu, Bridgewater, NJ, USA), the voltage from which was digitized using a high-speed data acquisition board (PXIe5185, National Instruments, Austin, TX, USA) operating with a temporal resolution of $80 \mathrm{ps}$. This enabled complete mapping of the sample surface in approximately $10 \mathrm{~min}$ at a resolution of $20 \mu \mathrm{m} /$ pixel. The mean and standard deviation of fluorescence lifetime in each spectral band were extracted from the FLIm images by defining circular regions of interest centered on the sample that encompassed $>75 \%$ of the surface area. The significant attenuation of $355 \mathrm{~nm}$ light in tissue limits the penetration depth of FLIm to 250-300 $\mu \mathrm{m}$ below the sample surface. To determine the translatability of the technique, viable samples were cut and scanned in cross-section to image depth-dependent changes in fluorescence LT with enzymatic degradation.

\section{TRFS system}

For TRFS measurements, a switchable mirror was used to direct the sample fluorescence to a monochromator, which was used to sequentially scan through the full fluorescence spectrum (360$600 \mathrm{~nm}$ at $5 \mathrm{~nm}$ increments). A PMT at the exit of 
the monochromator (MicroHR, Horiba Jobin Yvon, Edison, NJ, USA) was used to record the temporal dynamics of fluorescence at each wavelength. The PMT output was digitized using the same high-speed data acquisition board as the FLIm system. Five distinct points on the sample surface were measured and averaged at each wavelength. The mechanical scanning of the monochromator grating resulted in an acquisition time for TRFS of $30 \mathrm{~s}$ per sample.

\section{Biochemical analysis and histology}

For biochemical analysis, tissue samples were first measured to obtain wet weights ( $\mathrm{ww}$ ), lyophilized and reweighed to obtain dry weights (dw). Lyophilized samples were digested in papain solution for $18 \mathrm{~h}$ at $60{ }^{\circ} \mathrm{C}$, as previously described (Kochiadakis et al., 2001). Sulfated GAG content was assayed using the Blyscan ${ }^{\mathrm{TM}}$ Sulfated Glycosaminoglycan Assay kit (Biocolor, Westbury, NY, USA). Total collagen content was quantified using a chloramine-T hydroxyproline assay (Biocolor) (Woessner, 1961). For histological evaluation, samples were fixed in $10 \%$ neutral buffered formalin, paraffin-embedded and sectioned at $10 \mu \mathrm{m}$ thickness. Sections were stained with hematoxylin and eosin for general morphology, safranin $\mathrm{O}$ for glycosaminoglycans or picrosirius red for total collagen following routine procedures. Picrosirius-red-stained collagen was visualized under polarized light microscopy to enhance natural birefringence (Junqueira et al., 1979; Puchtler et al., 1973).

\section{Mechanical testing}

Compressive testing was performed on a $2 \mathrm{~mm}$ cylindrical punch from each sample, as previously described (Allen and Athanasiou, 2006). Briefly, after height detection was accomplished, a stress relaxation test was performed under conditions of unconfined compression. Samples were equilibrated in a PBS bath, pre-loaded to $0.02 \mathrm{~N}$ to ensure contact and preconditioned for 15 cycles of $5 \%$ strain. Next, they were placed under $10 \%$ strain for $600 \mathrm{~s}$ and $20 \%$ strain for $900 \mathrm{~s}$. Stress-strain data were analyzed using linear viscoelasticity theory (Mak et al., 1987) in Matlab v2018a (MathWorks, Inc., Natick, MA, USA) to calculate instantaneous modulus, relaxation modulus and coefficient of viscosity for each sample.

Tensile testing was conducted using a uniaxial materials testing machine (Test Resources, Shakopee, MN, USA), as previously described (Makris et al., 2013). Briefly, each articular cartilage sample was cut into a dog-bone shaped tensile specimen, photographed (Canon EOS 7D camera) and thickness and width were measured using ImageJ software (NIH) (Schindelin et al., 2015). A uniaxial strain to failure test was conducted at a strain rate of $1 \%$ of the gauge length/s. Young's modulus was calculated by least squares fitting the linear portion of the resulting stress-strain curve in Matlab.

\section{Statistical analysis and modeling}

All evaluations were performed using $n=6$ samples per treatment group. Data are presented as mean \pm standard deviation. Statistical analysis was performed using one-way ANOVA with Tukey's post hoc analysis. Significant differences $(p<0.05)$ are indicated by bars not sharing the same letter. For comparison between samples pre- and post-freeze, a matched paired $t$-test was used and significant differences $(p<0.05)$ are indicated by an asterisk.

Correlations between bivariate parameters of each enzymatic treatment were modeled separately to determine which outcome parameter correlated with which biochemical component using simple linear least squares regression analysis. Multivariable linear regression modeling was performed on the entire data set to determine the strength of the effect that multiple independent variables had on a single dependent outcome measurement. To determine which ECM components had significant effects on mechanical and imaging properties, collagen and GAG were modeled as the explanatory variables against $20 \%$ relaxation modulus and Young's modulus (Fig. 5) or average lifetimes from FLIm and TRFS (Fig. 6), respectively. To determine which mechanical properties had significant effects on the imaging properties, $20 \%$ relaxation modulus and Young's modulus were modeled as the explanatory variables against average lifetimes from FLIm and TRFS (Fig. 7). The predicted response (solid black line) and the $95 \%$ confidence interval (red dotted curves) for the predicted values are shown for each regression model (Fig. 5-7). To determine the predictive capabilities of the optical system in determining biochemical and mechanical properties, leave-one-out cross validation was performed on all linear models and root mean square error (RMSE) was calculated to determine the error associated with each model (Martens and Dardenne, 1998). All statistical analyses were performed on JMP v12 (SAS Institute, Cary, NC, USA) and cross validation was performed using Matlab.

\section{Results}

FLIm-derived parameters correlated with collagen depletion in articular cartilage

Nondestructive imaging of the articular surface of ColG-treated samples resulted in an apparent reduction in FLIm $\mathrm{CH} 1 \mathrm{LT}$ with increasing concentrations of ColG (Fig. 2a; representative scan). Mean fluorescence LTs in $\mathrm{CH} 2$ and $\mathrm{CH} 3$ were not significantly altered by ColG treatments $(p=0.78$ and 0.67 , respectively). Thus, only data from FLIm CH1 LT (termed FLIm LT) are presented. The FLIm LT signal within each sample was homogeneous and no voids or defects were detected in any of the samples tested. Quantification of the central region of 
interest of ColG-treated samples showed significant reductions in FLIm LT values with increased ColG treatment (Fig. 2b; $p=0.002$ ).

Collagenase treatment reduced both biochemical content and mechanical properties of articular cartilage

Biochemically, quantification of ColG-treated samples revealed a significantly decreased collagen and GAG content (Fig. 2c, $p=0.004$ and $p<0.001$, respectively). Collagenase treatment resulted in surface erosion, decreasing both collagen and GAG content, as seen qualitatively through picrosirius red and safranin $\mathrm{O}$ staining, respectively (Fig. 2d). Mechanically, both compressive and tensile properties significantly decreased with increased ColG treatment (Fig. 2e, $20 \%$ relaxation modulus, $p<0.0001$; Fig. 2 f, Young's modulus, $p=0.002$ ). Similarly, both instantaneous modulus and coefficient of viscosity significantly decreased with increased ColG treatment (Table 1 , both $p<0.0001$ ). Collagenase treatment had a significant, dose-dependent decrease in sample hydration (Table $1, p=0.001$ ).

TRFS-derived parameters correlated with proteoglycan depletion in articular cartilage

TRFS measurements of the articular surface of cABCtreated samples detected a significant decrease in

\section{Collagen Depletion}

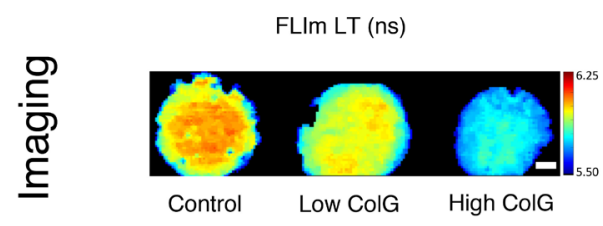

C
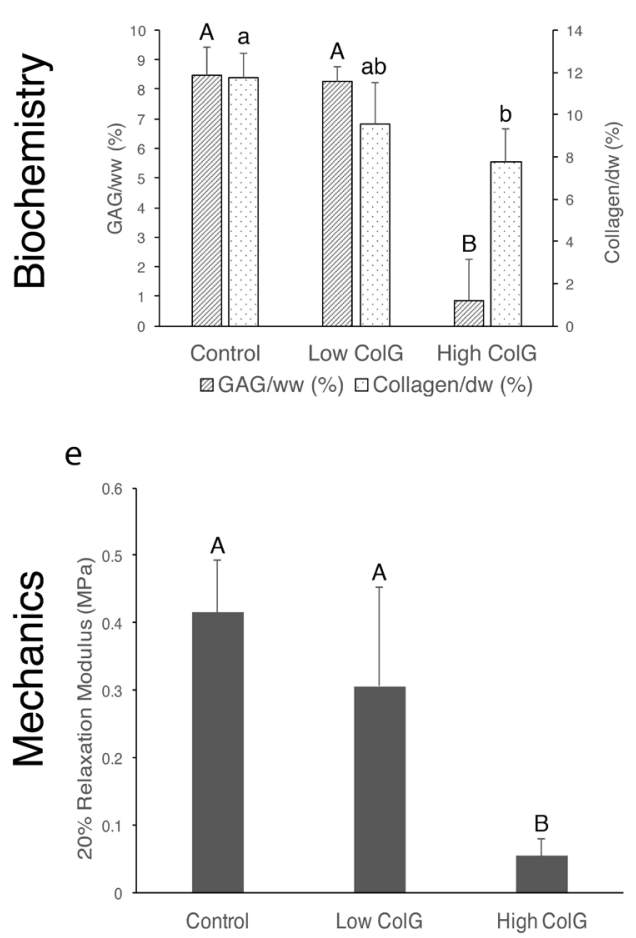

b

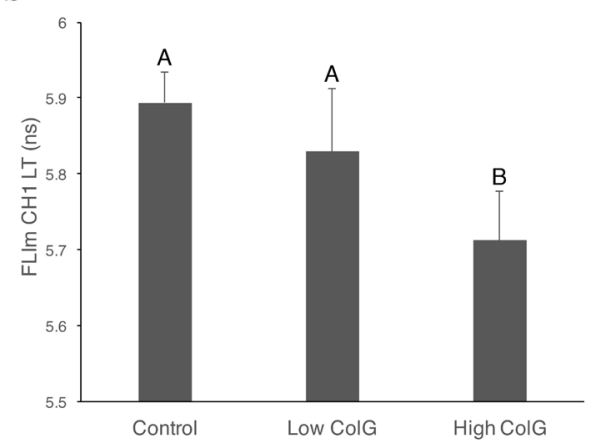

d

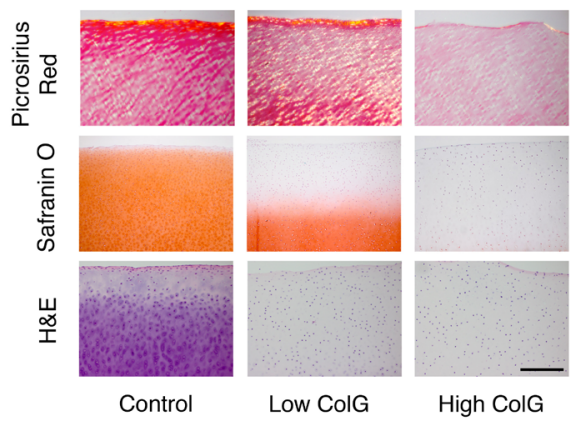

$f$

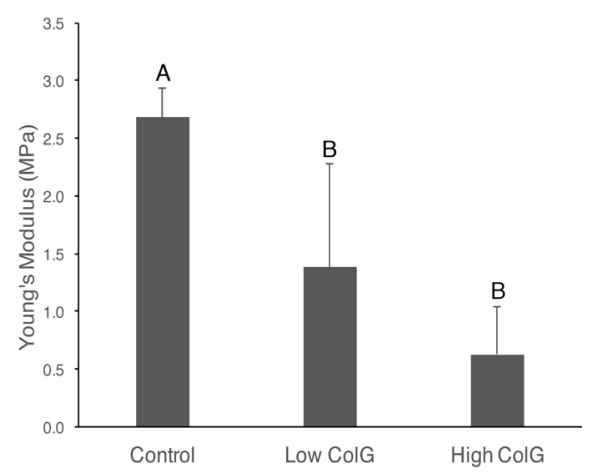

Fig. 2. FLIm-derived parameters correlated with collagen depletion in articular cartilage. (a) Representative FLIm scan of control, low ColG and high ColG corresponding to 0,20 and $80 \mu \mathrm{g} / \mathrm{mL}$ collagenase treatments (scale bar $=1 \mathrm{~mm}$ ). (b) Average FLIm LT decreased with collagenase treatment. (c) Collagen and GAG content decreased with collagenase treatment. (d) Picrosirius red and safranin O staining decreased with collagenase treatment. Hematoxylin and eosin staining showed morphological changes (scale bar $=200 \mu \mathrm{m}$ ). (e) Compressive modulus and (f) tensile modulus decreased with collagenase treatment. 
Table 1. Effects of enzymatic treatments on articular cartilage dry weight (DW), wet weight (WW), hydration, instantaneous modulus and coefficient of viscosity (mean \pm standard deviation).

\begin{tabular}{|l|c|c|c|c|c|}
\hline Treatment & DW (mg) & WW (mg) & Hydration (\%) & $\begin{array}{c}\text { 20 \% instantaneous } \\
\text { modulus (MPa) }\end{array}$ & $\begin{array}{c}\text { Coefficient of } \\
\text { viscosity (MPa } \times \text { s) }\end{array}$ \\
\hline Control & $1.3 \pm 0.2$ & $6.0 \pm 0.7$ & $63.6 \pm 5.6$ & $6.1 \pm 0.9$ & $53.0 \pm 3.9$ \\
\hline Low ColG & $1.7 \pm 0.2$ & $6.3 \pm 0.6$ & $58.1 \pm 9.0$ & $5.9 \pm 1.3$ & $36.2 \pm 7.9$ \\
\hline High ColG & $1.6 \pm 0.4$ & $6.2 \pm 1.3$ & $49.3 \pm 8.0$ & $1.5 \pm 1.1$ & $4.4 \pm 4.5$ \\
\hline Low cABC & $1.5 \pm 0.5$ & $5.9 \pm 0.9$ & $72.0 \pm 3.5$ & $1.2 \pm 0.9$ & $5.1 \pm 4.7$ \\
\hline High cABC & $0.9 \pm 2.8$ & $2.8 \pm 1.1$ & $74.1 \pm 6.5$ & $0.8 \pm 0.4$ & $3.2 \pm 2.1$ \\
\hline
\end{tabular}

\section{Proteoglycan Depletion}
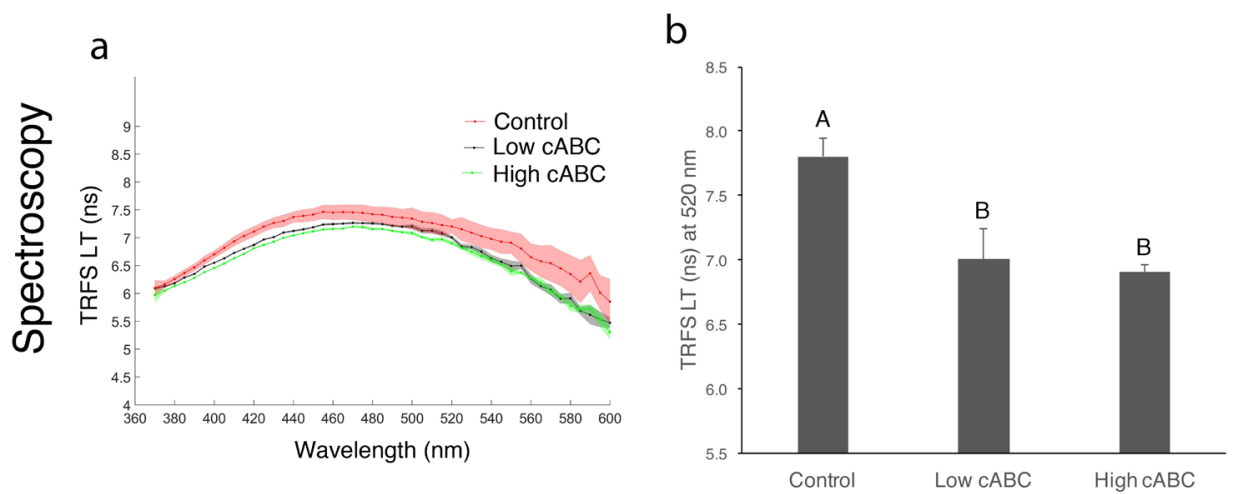

C

\section{d}
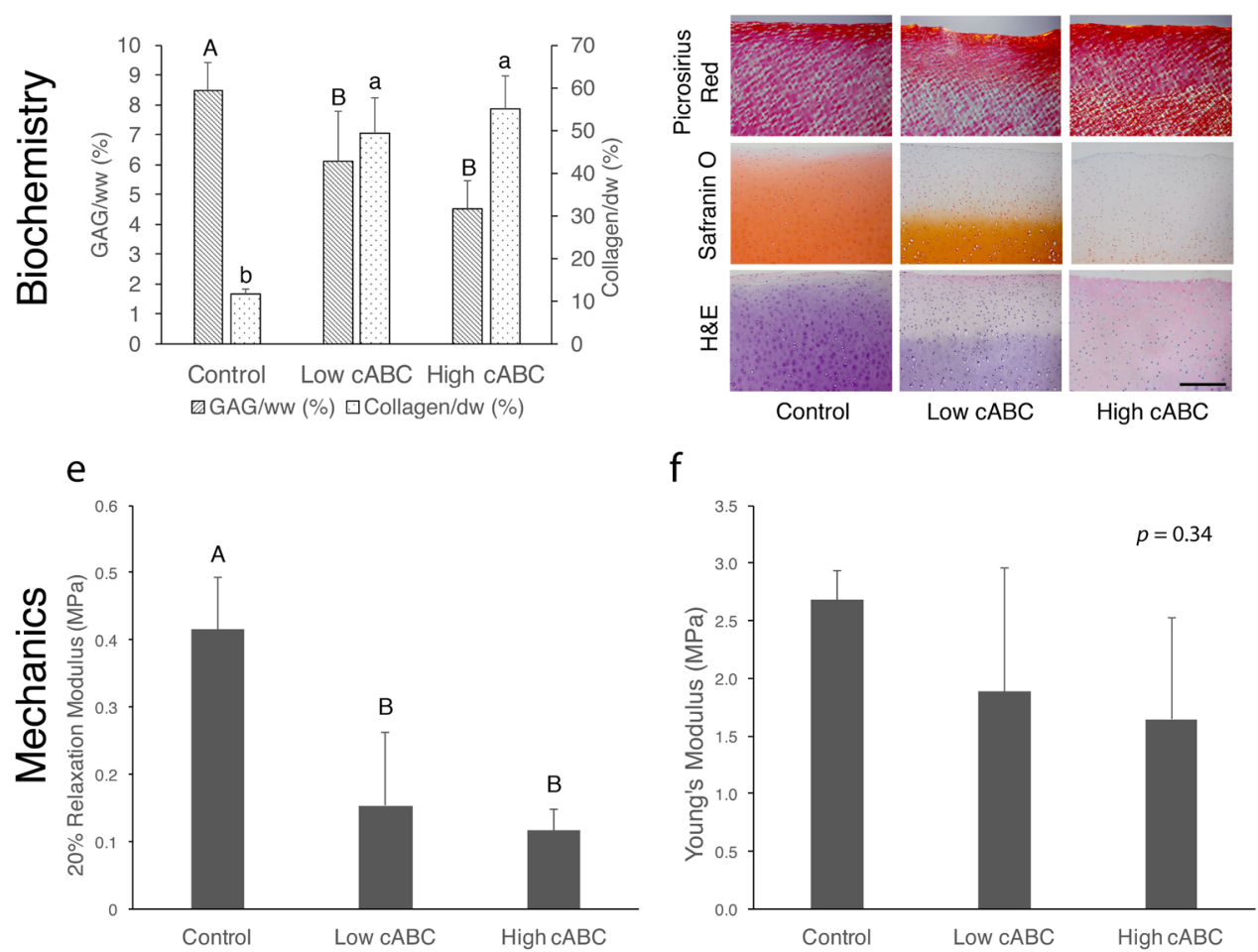

Fig. 3. TRFS-derived parameters correlated with proteoglycan depletion in articular cartilage. (a) Representative TRFS scan of control, low $\mathrm{CABC}$ and high cABC corresponding to $0,0.5$ and $1 \mathrm{U} / \mathrm{mL}$ cABC treatments. (b) TRFS LT at $520 \mathrm{~nm}$ decreased with collagenase treatment. (c) Collagen content increased and GAG content decreased with cABC treatment. (d) With cABC treatment, picrosirius red increased, safranin $O$ staining decreased and hematoxylin and eosin staining showed morphological changes (scale bar $=200 \mu \mathrm{m}$ ). (e) Compressive modulus decreased whereas (f) tensile modulus did not significantly change with $\mathrm{CABC}$ treatment. 
lifetime in the $520 \pm 5 \mathrm{~nm}$ region of the spectrum. Thus, only data from TRFS LT signal at $520 \pm 5 \mathrm{~nm}$ (termed TRFS LT) are presented. The reduction in TRFS LT correlated with increased concentrations of cABC (Fig. 3a; representative image). Quantification of TRFS LT in cABC-treated samples showed significant reductions in TRFS LT values with increased CABC treatment (Fig. 3b; $p<0.001$ ).

cABC treatment altered both biochemical content and compressive properties of articular cartilage Biochemically, quantification of cABC-treated samples showed a significant increase in collagen content and a decrease in GAG content with increased cABC concentrations (Fig. 3c, $p=0.004$ and $p<0.001$, respectively). $c A B C$ treatment resulted in surface erosion of GAG, as seen qualitatively through a decreased safranin $\mathrm{O}$ staining, and an increase in collagen staining, as observed in picrosirius red staining (Fig. 3d). Mechanically, compressive properties significantly decreased with increased cABC treatment (Fig. 3e, $20 \%$ relaxation modulus, $p<0.0001)$ while tensile properties were not significantly affected by either low or high $\mathrm{cABC}$ treatment (Fig. 3f, Young's modulus, $p=0.34$ ). Similarly, both the instantaneous modulus and the coefficient of viscosity significantly decreased with increased $\mathrm{CABC}$ treatment (Table 1 , both $p<0.0001$ ).
cABC treatment resulted in a significant, dosedependent increase in sample hydration (Table 1, $p=0.03)$.

Combined FLIm- and TRFS-derived parameters were sensitive to changes in cartilage matrix and correlated with destructive mechanical and biochemical assays

Bivariate analysis of ColG-treated samples revealed significant, linear correlations between FLIM LT and collagen content (Fig. $4 \mathbf{a}, R^{2}=0.79, p<0.001$ ) and between FLIM LT and Young's modulus (Fig. 4b, $\left.R^{2}=0.45, p=0.02\right)$. Cross-validation of FLIm-based predictions of collagen content and Young's modulus determined the RMSE to be 1.15 and 0.9 , respectively.

Bivariate analysis of cABC-treated samples revealed significant, linear correlations between TRFS LT and GAG content (Fig. 4c, $R^{2}=0.69, p=0.001$ ) and between TRFS LT and compressive modulus (Fig. $\left.4 \mathrm{~d}, R^{2}=0.76, p<0.001\right)$. Cross-validation of TRFSbased predictions of GAG content and compressive modulus determined the RMSE to be 1.24 and 0.09 , respectively.

\section{Mechanical properties correlated with biochemical content in cartilage}

A multivariable linear regression model of all biochemical data against tensile data showed
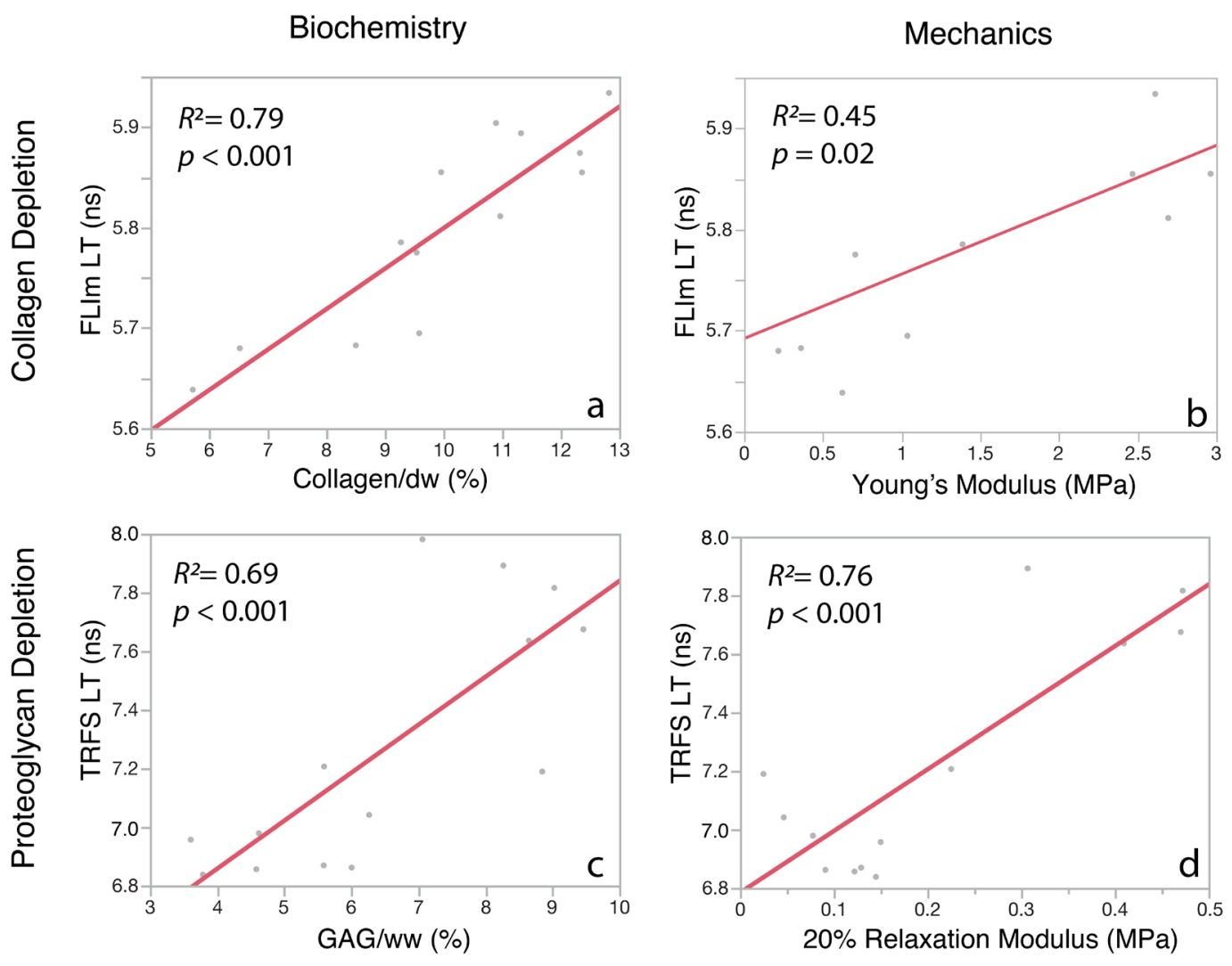

Fig. 4. FLIm- and TRFS-derived parameters correlated with biochemical and mechanical properties in articular cartilage. In collagen-depleted samples, FLIm LT significantly correlated with (a) collagen content and (b) tensile Young's modulus. In proteoglycan-depleted samples, TRFS LT significantly correlated with (c) proteoglycan content and (d) compressive relaxation modulus. 
that GAG content was not a significant effect in determining Young's modulus (Fig. 5a; $p=0.84$ ). However, increased collagen content significantly increased the Young's modulus (Fig. 5b; $p=0.01$ ). A multivariable linear regression model of all biochemical data against compression data showed that increased GAG content significantly increased compressive modulus (Fig. 5c; $p=0.002$ ), but increased collagen content was not a significant effect in determining compressive modulus (Fig. 5d; $p=0.38)$.

\section{FLIm-derived parameters correlated with destructive biochemical testing in cartilage}

A multivariable linear regression model of all biochemical testing data against FLIm data showed that GAG content was not a significant effect in determining FLIm LT values (Fig. 6a; $p=0.90$ ), but increased collagen content significantly increased FLIm LT values (Fig. 6b; $p<0.01$ ). A multivariable linear regression model of all destructive biochemical testing data against TRFS data showed that increased GAG content significantly increased TRFS LT values (Fig. 6c; $p=0.004$ ), but was not a significant effect in determining TRFS LT (Fig. $6 \mathbf{d} ; p=0.60$ ).
FLIm- and TRFS-derived parameters correlated with mechanical properties in cartilage

A multivariable linear regression model of mechanical testing data against FLIm measurements showed that compressive modulus was not a significant effect in determining FLIm LT values (Fig. 7a; $p=0.48$ ) but increased tensile modulus significantly altered FLIm LT (Fig. 7b; $p=0.02$ ). A multivariable linear regression model of mechanical testing data against TRFS parameters showed that increased compressive modulus but not increased tensile modulus were significant effects in determining TRFS LT values (Fig. 7c, $p=0.003$ and Fig. $7 d, p=0.75$, respectively).

Freeze-thaw processing produced wavelength specific changes in fluorescence LT in articular cartilage

Freeze-thaw processing resulted in wavelength specific changes in fluorescence LT [Fig. 8a; representative images of viable $(\mathrm{V})$ and frozen $(\mathrm{F})$ samples]. Quantification of FLIm LT showed that the freeze-thaw processing resulted in a significant decrease in FLIm CH1 LT (Fig. 8b; $p=0.003 ; \Delta=0.14$ ) and a significant increase in FLIm CH2 LT (Fig. $8 \mathbf{b} ; p=0.003 ; \Delta=0.11$ ) and FLIm CH3 LT (Fig. 8b; $p=0.003 ; \Delta=0.22$ ) as compared to viable tissue.
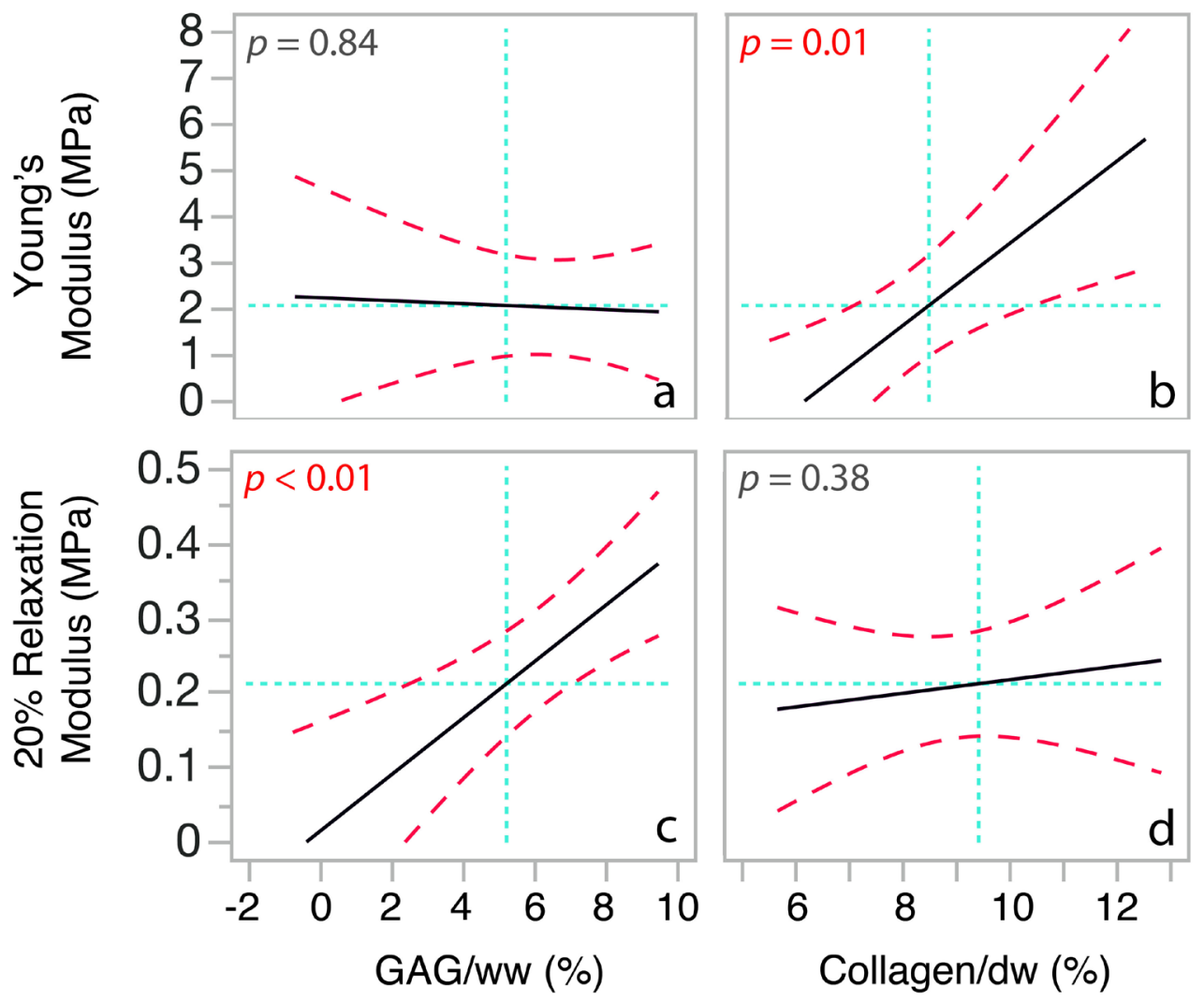

Fig. 5. Mechanical properties correlated with biochemical content. (a) GAG content was not a significant effect Young's modulus $(p=0.84)$, while $(\mathbf{b})$ increased collagen content significantly increased Young's Modulus $(p=0.01)$. (c) Increased GAG content significantly increased compressive modulus $(p<0.01)$, while (d) increased collagen content was not a significant effect in determining compressive modulus $(p=0.38)$. 

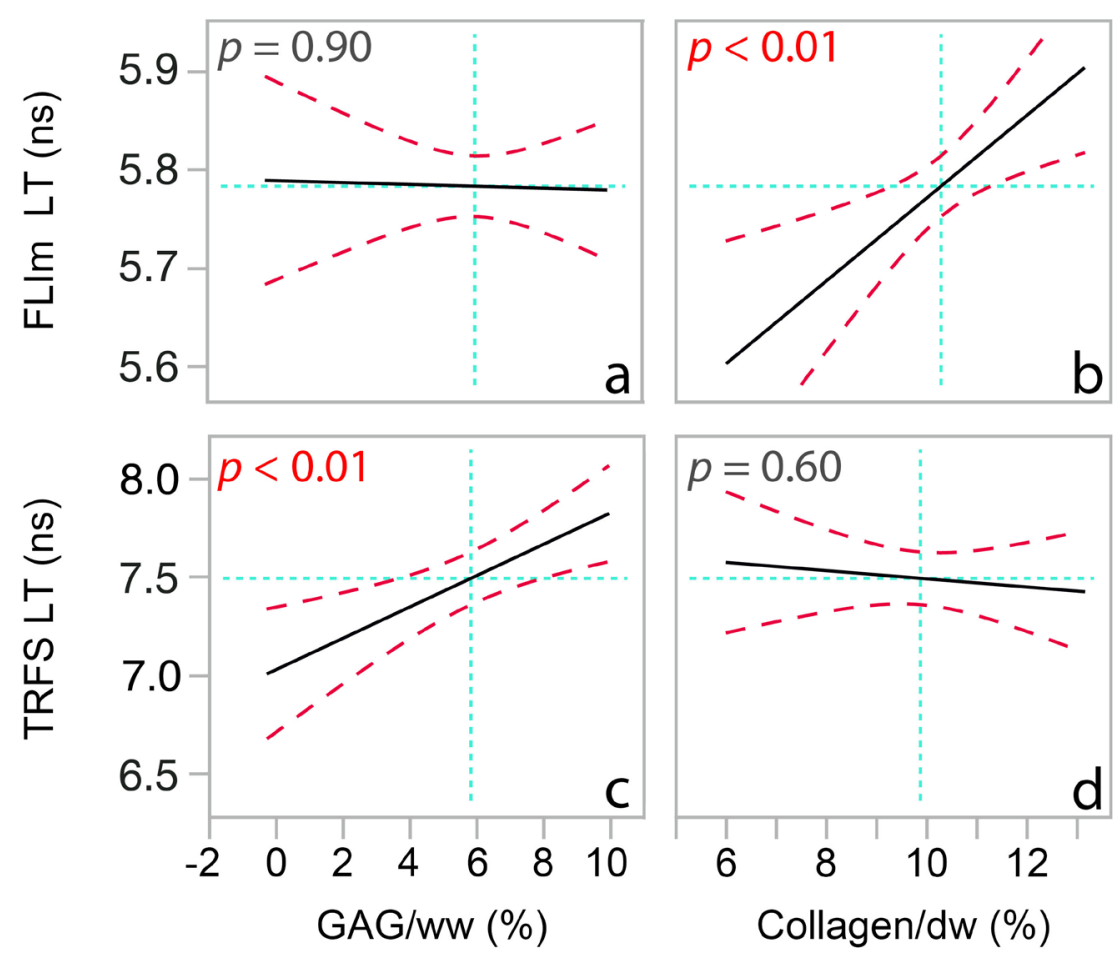

Fig. 6. FLIm- and TRFS-derived parameters correlated with destructive biochemical testing. (a) GAG content was not a significant effect in determining FLIm LT values $(p=0.90)$, but $(\mathbf{b})$ increased collagen content significantly increased FLIm LT values $(p<0.01)$. (c) Increased GAG content significantly increased TRFS LT values $(p<0.01)$, while $(\mathbf{d})$ increased collagen content was not a significant effect in determining TRFS LT values $(p=0.60)$.
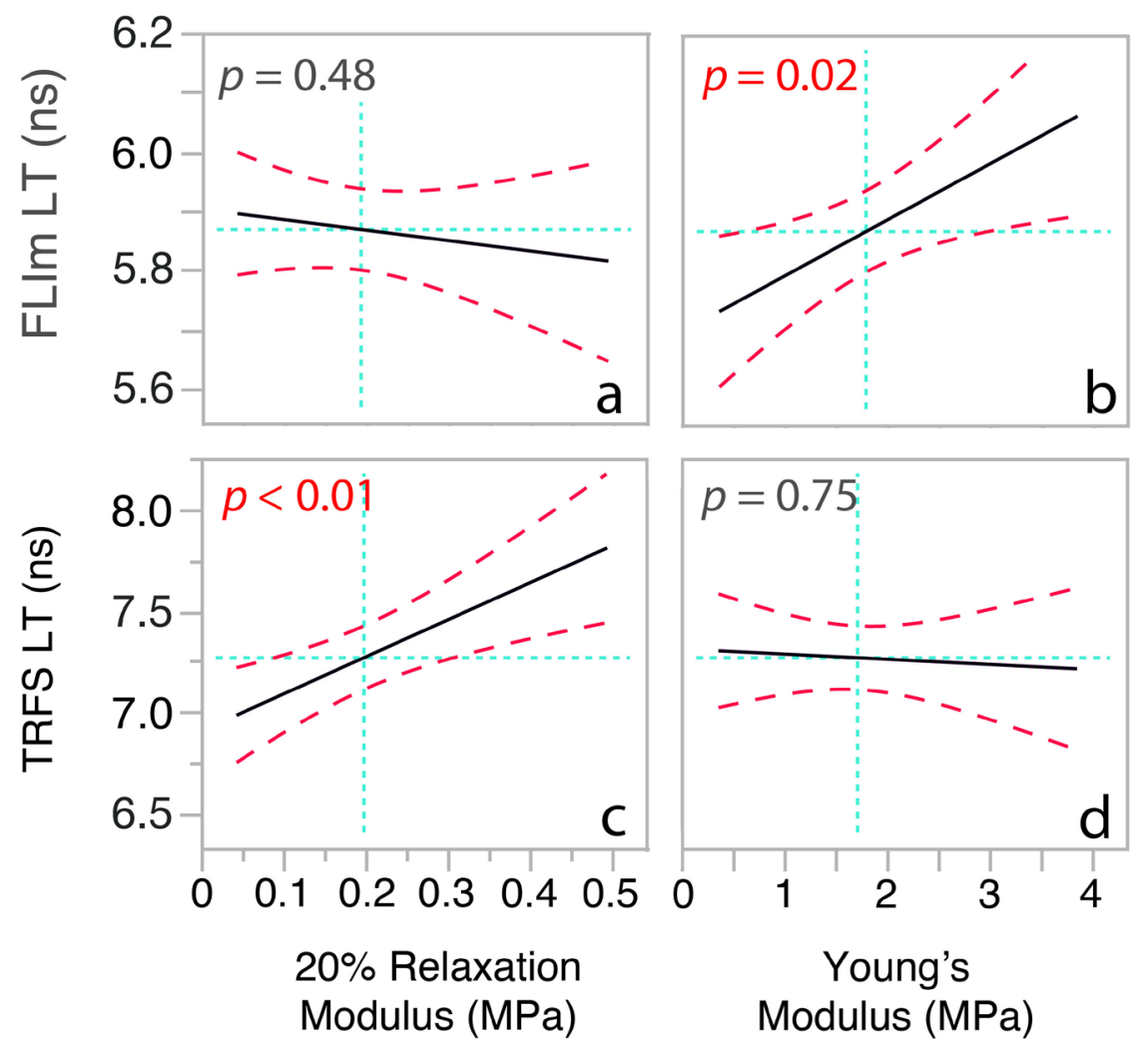

Fig. 7. Optical parameters correlated with mechanical properties in articular cartilage. (a) Compressive relaxation modulus was not a significant effect in determining FLIm LT values $(p=0.48)$, but $(\mathbf{b})$ increased tensile Young's modulus significantly affected FLIm LT values $(p=0.02)$. (c) Increased compressive relaxation modulus significantly affected TRFS LT values $(p<0.01)$, while $(\mathbf{d})$ increased tensile Young's modulus was not a significant effect in determining TRFS LT values $(p=0.75)$. 
Cross-sectional FLIm imaging of viable cartilage showed depth-dependent changes in enzymedepleted articular cartilage

Cross-sectional FLIm assessment of viable articular cartilage samples qualitatively showed decreased FLIm CH1 LT at the surface of ColG-treated samples and decreased FLIm CH3 LT at the surface of CABCtreated samples as compared to control (Fig. 8c and $8 d$, respectively). Decreased FLIm CH1 LT signal correlated with areas of reduced picrosirius red staining in ColG-treated samples with respect to control samples (Fig. 8c; denoted by $\ddagger$ ). Similarly, decreased FLIm CH3 LT signal correlated with areas of reduced safranin $\mathrm{O}$ staining in $\mathrm{CABC}$-treated sample with respect to control samples (Fig. 8d; denoted by an asterisk).

\section{Discussion}

The results of the present study demonstrated the applicability of using a combination of nondestructive imaging and spectroscopic techniques to determine collagen and proteoglycan content and changes in the tensile and compressive properties of articular cartilage that occur with matrix depletion. The hypothesis that noninvasive assessments could be used to infer the changes in both the biochemical composition and biomechanical properties of cartilage that occur during matrix depletion was supported by the strong linear correlations between destructive and nondestructive tests, making this the first investigation that demonstrated quantitative relationships between fluorescence LT and articular cartilage mechanical properties. The novel findings of this experiment included: 1) quantitative relationships between TRFS LT and both proteoglycan content and compressive moduli and 2) quantitative relationships between FLIm LT and collagen content and tensile modulus. The results of this study are particularly exciting because they can serve as an initial step toward the creation of a nondestructive diagnostic tool for articular cartilage.

The use of enzymatic treatments allowed two objectives to be achieved: 1) the enzymatic treatments mimicked aspects of cartilage degradation under pathology and 2) the treatments yielded samples with varying levels of matrix contents and mechanical properties. Decreases in collagen and GAG content are seen in the early development of osteoarthritis
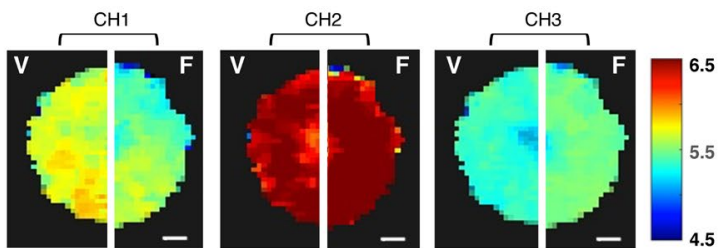

b

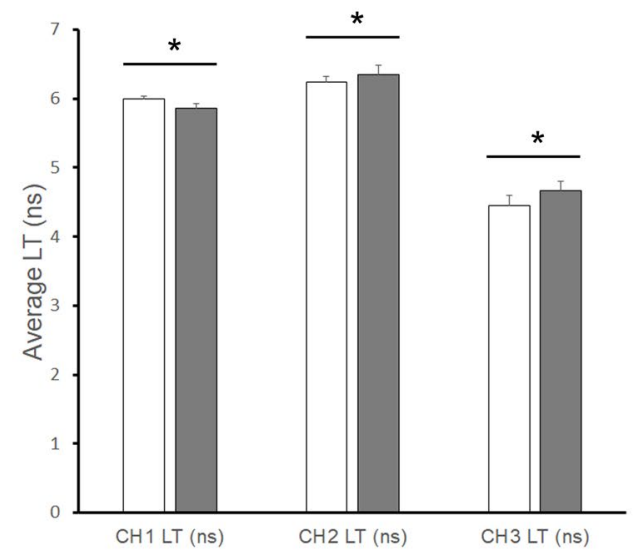

C
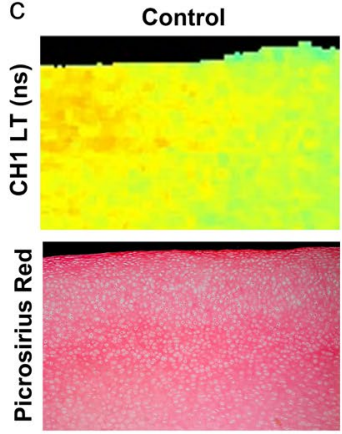

d

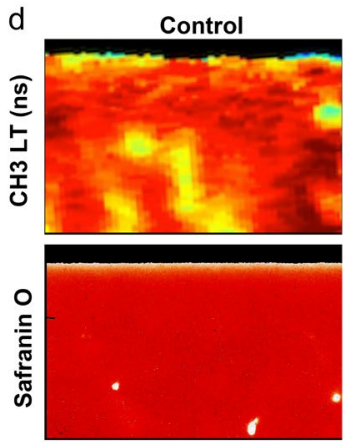

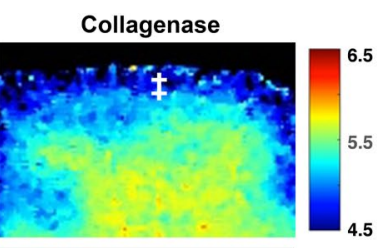

$\ddagger$
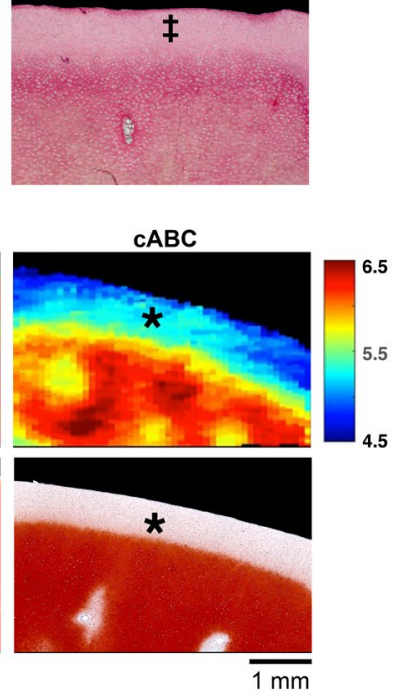

Fig. 8. Comparison between fluorescence LT of viable and freeze-thaw-processed articular cartilage. (a) Representative FLIm images of viable (V) articular cartilage and after freeze-thaw processing (F) in each of the three spectral bands (scale $=1 \mathrm{~mm})$. (b) Quantification of FLIm LT showed that the freeze-thaw processing (grey bar) resulted in a slight but significant decrease in FLIm CH1 LT $(p=0.003 ; \Delta=0.14)$ and a significant increase in FLIm CH2 LT $(p=0.003 ; \Delta=0.11)$ and FLIm CH3 LT $(p=0.003 ; \Delta=0.22)$ as compared to viable tissue (white bar). (c) Cross-sectional fluorescence LT image (CH1: 375-410 nm spectral band) of ColGdegraded articular cartilage showed decreased LT in collagen-depleted regions $(\ddagger)$ that corresponded to less-stained regions in picrosirius red histological sections. (d) Cross-sectional fluorescence LT image (CH3: 532-565 nm spectral band) of cABC-degraded articular cartilage showed decreased LT in GAG-depleted regions $\left({ }^{*}\right)$ that corresponded to unstained regions in safranin $\mathrm{O}$ histological sections (scale bar $=1 \mathrm{~mm}$ ). 
in articular cartilage (Squires et al., 2003). Similarly, the decrease in cartilage ECM composition created by the enzymatic treatments employed in this study is well established. Collagen and GAG content in articular cartilage treated with the highest dose of ColG were reduced to $66 \%$ and $10 \%$, respectively, of untreated controls. The reduction in GAG content after ColG treatment was likely due to the inability of matrix with digested collagen to retain the highly charged GAG matrix within the tissue. In CABCtreated cartilage, GAG content was reduced to $53 \%$ of its original value, as similarly reported by Agemura et al. (1990). Utilization of both enzymatic treatments caused dose-dependent reductions in matrix components from articular cartilage, creating a range of biochemical and mechanical properties for optical characterization.

The present study reaffirmed the structurefunction relationships within articular cartilage, where its material properties are determined by the biochemical composition of the main tissue constituents, GAG and collagen (Setton et al., 1993). Multivariable linear regression models evaluating the effects of collagen content or GAG content showed that the collagen matrix content significantly regulated the tensile response of the cartilage $(p=0.01)$. A high dose of ColG resulted in a 5-fold decrease in tensile properties. Similarly, multivariable linear regression models found that proteoglycan matrix content significantly affected the compressive response of the cartilage $(p<0.01)$. A high dose of $\mathrm{CABC}$ resulted in a 4 -fold decrease in compressive properties. Based on these and prior literature findings (Pritzker et al., 2006), it is expected that changes in cartilage composition and structure, including the depletion of the collagen network and GAGs, result in the impaired functional integrity of the cartilage.

A combination of TRFS and FLIm in a single apparatus was capable of characterizing the identity, quantity and distribution of both collagen and proteoglycans in articular cartilage and of forming quantitative relationships with tissue mechanical properties. The strong correlations between TRFS LT and GAG content $\left(R^{2}=0.59 ; p=0.001\right)$ and between FLIm LT and collagen content $\left(R^{2}=0.79 ; p<0.001\right)$ support the use of nondestructive techniques for the quantification of the cartilage biochemical components. While fluorescence lifetime techniques are sensitive to changes in sample biochemistry and do not directly measure tissue mechanical properties, the strong structure-function relationship resulted in strong correlations between each optical assessment technique and specific mechanical properties. TRFS LT had a strong correlation with tissue compressive relaxation modulus $\left(R^{2}=0.71 ; p<0.001\right)$. Likewise, FLIm LT resulted in a significant correlation with tensile Young's modulus $\left(R^{2}=0.45 ; p=0.02\right)$. During cABC treatment, GAG removal relatively enriched the collagen content with respect to native tissue values. While the relative collagen content increased 4-fold, the tensile properties were not significantly improved; thus, the optical measurements are not just coincidentally related to the mechanical properties, but also dependent upon ECM structure (Morgan et al., 2014). Because optical properties are attributed not only to the number of molecules present but also to their environment (Marcu et al., 2014), the imaging probe measurements provide more of bulk property of the tissue. By encasing both optical techniques in one probe, it is possible to envision a single, nondestructive tool for measuring both biological content and mechanical properties.

The combination of TRFS and FLIm in a single apparatus exploited the natural complementarity that exists between the two techniques. FLIm is a high-throughput technique that is designed to record the spatial distribution of endogenous fluorophores at high spatial sampling (Marcu et al., 2014). FLIm images typically contain several thousand pixels, allowing the use of statistical methods to detect subtle shifts in fluorescence lifetime. The primary drawback of FLIm is its limited spectral resolution, which reduces its sensitivity to changes in sample fluorescence that are only apparent within narrow regions of the spectrum. TRFS is a point measurement technique that records sample autofluorescence at high spectral resolution (Marcu et al., 2014). Although TRFS does not capture spatial information, a highresolution measurement of the fluorescence spectrum can identify the spectral regions that provide optimal contrast between groups that other methods cannot detect. This feature could be exploited in the design of future FLIm systems, where the distribution of spectral bands could be determined by TRFS measurements of a particular tissue type. In the case of a dedicated cartilage FLIm system, it is possible to envision designing a custom apparatus with two spectral bands, centered at $390 \pm 10 \mathrm{~nm}$ and $520 \pm 5 \mathrm{~nm}$, for detecting changes in collagen and GAG, respectively.

A range of alternative optical techniques have previously been employed to monitor changes in the structure and biochemistry of articular cartilage. Fourier transform infrared (FT-IR) spectroscopy is successfully used to measure differences in the composition of healthy and osteoarthritic cartilage (Rieppo et al., 2012). While FT-IR can be employed with fiber optics in reflection mode, the method requires a constant level of contact with the tissue sample to produce reliable results and its large probe size limits both in vivo access to the joint as well as spatial resolution (Hanifi et al., 2012). These technical challenges need to be resolved before this imaging technique could be readily deployed in vivo. Raman spectroscopy can delineate cartilage zonal structure (Bergholt et al., 2016) and detect lipid distribution (Mansfield and Winlove, 2017). While Raman spectroscopy generally utilizes longer excitation wavelengths than $355 \mathrm{~nm}$, resulting in greater penetration depths, it is an inherently slow technique with individual spectra often taking between 0.1- 
$0.5 \mathrm{~s} /$ acquisition (Bergholt et al., 2016). By comparison, FLIm and TRFS acquired complete fluorescent decays imaging data in $<10 \mathrm{~ms}$. The order of magnitude difference in image acquisition speed is critical with respect to the translational potential of FLIm and TRFS over Raman-based approaches. Finally, optical coherence tomography (OCT) is widely used to image articular cartilage microstructure (Nebelung et al., 2015) and is successfully deployed arthroscopically (Liukkonen et al., 2013). This highspeed, high-resolution imaging technique benefits from the enhanced penetration depths afforded by near-infrared light, allowing OCT to resolve cartilage structure between 1-2 mm below the surface. Despite several encouraging developments, OCT lacks biochemical sensitivity and it cannot reliably detect changes in proteoglycan content (Saarakkala et al., 2009). In comparison, the fiber-based FLIm-TRFS imaging platform is designed to rapidly acquire high-resolution images in real time and to ease the translation into human imaging by augmentation of the current clinical practice of arthroscopy. By incorporating the FLIm fiber into a conventional viewing scope, this technique would enable straightforward visual evaluation of the integrity of the cartilage surface, as well as determination of quantitative parameters reflecting the quality of articular cartilage tissue. Furthermore, decreasing the diameter of the optical-probe fiber so that it can be inserted through a standard needle would open the potential for nondestructive evaluation of articular cartilage health as an office procedure. Use of such a minimally-invasive technique for evaluation of cartilage quality could facilitate early intervention and assess the efficacy of rehabilitation programs and therapeutic interventions on cartilage repair.

While the present study focused on the nondestructive evaluation of native cartilage depletion ex vivo, these techniques could be employed to execute longitudinal research studies, create more efficient animal studies requiring fewer animal sacrifices, monitor product development in an industrial manufacturing process and quantify clinically cartilage degeneration and repair. The optical system was able to measure changes in articular cartilage composition from scans made perpendicular to the articular surface toward the bone. While this scan direction is the most relevant clinically, there is a limited penetration depth of the system (200-250 $\mu \mathrm{m}$ at wavelengths of $355 \mathrm{~nm})$. That, coupled with the surface erosion of the proteins in the tissue subjected to enzymatic treatments, resulted in lower than expected differences in fluorescence signal. Larger changes in signal are possible by switching to multiphoton excitation of fluorescence (Sherlock et al., 2016). This technique provides intrinsic optical sectioning, confining the extent of the region within which fluorescence is excited to less than $10 \mu \mathrm{m}$. Multiphoton excitation of fluorescence has the potential to increase the overall sensitivity of the device, allowing the system to better discriminate between degraded and healthy cartilage regions.

Freeze-thaw cycles were used to remove the cellular component of the articular cartilage to avoid interference of the cells with imaging modalities. While freeze-thaw processing was able to isolate individual protein contributions to fluorescence LT, it did not fully represent conditions encountered in the final application of this method. Studies on freshlyharvested articular cartilage were conducted to determine whether the optical system could assess a viable tissue. While the freezing process did produce significant changes in fluorescence LT, the changes were small when compared to changes created by matrix degradation $(\Delta=1.0 \mathrm{~ns}$ for cABC-digestion $v s$. $\Delta=0.22 \mathrm{~ns}$ for freeze-thaw). These small differences in cellular contribution were most likely due to the low ( $10 \%$ by volume) composition of cells within articular cartilage. It is possible that the elimination of the low fluorescence LT signal of cells ( 2-3 ns) in $\mathrm{CH} 2$ and $\mathrm{CH} 3$ resulted in an increase in LT in both channels upon cell removal with freeze-thaw processing. The slight decrease in FLIm CH1 was likely due to the breaking of pyridinoline crosslinks by the expansion of intra-fibrillar fluid during ice formation in the freezing process (Ozcelikkale and Han, 2016). Matrix degradation by both ColG and cABC was detectable with FLIm assessment of viable articular cartilage samples and correlated well with histological staining, supporting the use of the optical system in a diagnostic application.

In conclusion, the label-free optical techniques FLIm and TRFS successfully detected collagen and proteoglycan changes in articular cartilage composition in both viable and previously frozen tissues. Using multivariable linear regression models, these complimentary optical techniques were found to be strongly correlated with compressive and tensile properties. Specifically, the FLIm LT optical system could detect changes in collagen content and tensile properties while the TRFS LT optical system could detect changes in GAG content and compressive properties of articular cartilage. The development of nondestructive tools with potential to monitor the functional properties of cartilage is an exciting step forward for both in vitro and in vivo studies in the future.

\section{Acknowledgements}

This work was funded by the California Institute for Regenerative Medicine (CIRM) grant RT3-07981. This funding source did not play any role in the collection, analysis or interpretation of the data presented. All authors conceived and designed this study: $\mathrm{AKH}, \mathrm{BES}$ and $\mathrm{XZ}$ acquired the data; $\mathrm{AKH}$ provided statistical analysis; all authors reviewed and interpreted the data, wrote and edited the manuscript 
and read and approved the final version. The authors have no conflicts of interest to declare.

\section{References}

Agemura DH, O'Brien WD, Jr., Olerud JE, Chun LE, Eyre DE (1990) Ultrasonic propagation properties of articular cartilage at $100 \mathrm{MHz}$. J Acoust Soc Am 87: 1786-1791.

Allen KD, Athanasiou KA (2006) Viscoelastic characterization of the porcine temporomandibular joint disc under unconfined compression. J Biomech 39: 312-322.

Bergholt MS, St-Pierre JP, Offeddu GS, Parmar PA, Albro MB, Puetzer JL, Oyen ML, Stevens MM (2016) Raman spectroscopy reveals new insights into the zonal organization of native and tissue-engineered articular cartilage. ACS Cent Sci 2: 885-895.

Chojkier M, Peterkofsky B, Bateman J (1980) New method for determining the extent of proline hydroxylation by measuring changes in the ratio of $[4-3 \mathrm{H}]:[14 \mathrm{C}]$ proline in collagenase digests. Anal Biochem 108: 385-393.

Fite BZ, Decaris M, Sun Y, Sun Y, Lam A, Ho CK, Leach JK, Marcu L (2011) Noninvasive multimodal evaluation of bioengineered cartilage constructs combining time-resolved fluorescence and ultrasound imaging. Tissue Eng Part C Methods 17: 495-504.

Georgakoudi I, Jacobson BC, Muller MG, Sheets EE, Badizadegan K, Carr-Locke DL, Crum CP, Boone CW, Dasari RR, Van Dam J, Feld MS (2002) NAD(P) $\mathrm{H}$ and collagen as in vivo quantitative fluorescent biomarkers of epithelial precancerous changes. Cancer Res 62: 682-687.

Gorpas D, Fatakdawala H, Bec J, Ma D, Yankelevich DR, Qi J, Marcu L (2015) Fluorescence lifetime imaging and intravascular ultrasound: co-registration study using ex vivo human coronaries. IEEE Trans Med Imaging 34: 156-166.

Gorpas D, Ma D, Bec J, Yankelevich DR, Marcu L (2016) Real-time visualization of tissue surface biochemical features derived from fluorescence lifetime measurements. IEEE Trans Med Imaging 35: 1802-1811.

Hamai A, Hashimoto N, Mochizuki H, Kato F, Makiguchi Y, Horie K, Suzuki S (1997) Two distinct chondroitin sulfate $A B C$ lyases. An endoeliminase yielding tetrasaccharides and an exoeliminase preferentially acting on oligosaccharides. J Biol Chem 272: 9123-9130.

Hanifi A, Bi X, Yang X, Kavukcuoglu B, Lin PC, DiCarlo E, Spencer RG, Bostrom MP, Pleshko N (2012) Infrared fiber optic probe evaluation of degenerative cartilage correlates to histological grading. Am J Sports Med 40: 2853-2861.

Huey DJ, Hu JC, Athanasiou KA (2012) Unlike bone, cartilage regeneration remains elusive. Science 338: 917-921.
Junqueira LCU, Bignolas G, Brentani RR (1979) Picrosirius staining plus polarization microscopy, a specific method for collagen detection in tissue sections. Histochem J 11: 447-455.

Kochiadakis GE, Chrysostomakis SI, Kalebubas MD, Filippidis GM, Zacharakis IG, Papazoglou TG, Vardas PE (2001) The role of laser-induced fluorescence in myocardial tissue characterization: an experimental in vitro study. Chest 120: 233-239.

Liu J, Sun Y, Qi J, Marcu L (2012) A novel method for fast and robust estimation of fluorescence decay dynamics using constrained least-squares deconvolution with Laguerre expansion. Phys Med Biol 57: 843-865.

Liukkonen J, Hirvasniemi J, Joukainen A, Penttila P, Viren T, Saarakkala S, Kroger H, Jurvelin JS, Toyras J (2013) Arthroscopic ultrasound technique for simultaneous quantitative assessment of articular cartilage and subchondral bone: an in vitro and in vivo feasibility study. Ultrasound Med Biol 39: 1460-1468.

Mak AF, Lai WM, Mow VC (1987) Biphasic indentation of articular cartilage-I. Theoretical analysis. J Biomech 20: 703-714.

Makris EA, Gomoll AH, Malizos KN, Hu JC, Athanasiou KA (2015) Repair and tissue engineering techniques for articular cartilage. Nat Rev Rheumatol 11: 21-34.

Makris EA, MacBarb RF, Responte DJ, Hu JC, Athanasiou KA (2013) A copper sulfate and hydroxylysine treatment regimen for enhancing collagen cross-linking and biomechanical properties in engineered neocartilage. FASEB J 27: 2421-2430.

Manning HB, Nickdel MB, Yamamoto K, Lagarto JL, Kelly DJ, Talbot CB, Kennedy G, Dudhia J, Lever J, Dunsby C, French P, Itoh Y (2013) Detection of cartilage matrix degradation by autofluorescence lifetime. Matrix Biol 32: 32-38.

Mansfield JC, Winlove CP (2017) Lipid distribution, composition and uptake in bovine articular cartilage studied using Raman micro-spectrometry and confocal microscopy. J Anat 231: 156-166.

Marcu L, French PMW, Elson DS (2014) Fluorescence lifetime spectroscopy and imaging: principles and applications in biomedical diagnostics. CRC Press/Taylor \& Francis Group, Boca Raton, FL, USA.

Martens HA, Dardenne P (1998) Validation and verification of regression in small data sets. Chemometr Intell Lab Syst 44: 99-121.

Morgan SP, Rose FR, Matcher SJ (2014) Optical techniques in regenerative medicine. Taylor \& Francis, Boca Raton, FL, USA.

Nebelung S, Brill N, Marx U, Quack V, Tingart M, Schmitt R, Rath B, Jahr H (2015) Three-dimensional imaging and analysis of human cartilage degeneration using optical coherence tomography. J Orthop Res 33: 651-659.

Ozcelikkale A, Han B (2016) Thermal destabilization of collagen matrix hierarchical structure by freeze/thaw. PLoS One 11: DOI: 10.1371/ journal.pone.0146660. 
Pritzker KPH, Gay S, Jimenez SA, Ostergaard K, Pelletier JP, Revell PA, Salter D, van den Berg WB (2006) Osteoarthritis cartilage histopathology: grading and staging. Osteoarthritis Cartilage 14: 1329.

Puchtler H, Waldrop FS, Valentine LS (1973) Polarization microscopic studies of connective tissue stained with picro-sirius red FBA. Beitr Pathol 150: 174-187.

Requejo-Isidro J, McGinty J, Munro I, Elson DS, Galletly NP, Lever MJ, Neil MAA, Stamp GWH, French PMW, Kellett PA, Hares JD, DymokeBradshaw AKL (2004) High-speed wide-field timegated endoscopic fluorescence-lifetime imaging. Optics Letters 29: 2249-2251.

Responte DJ, Natoli RM, Athanasiou KA (2007) Collagens of articular cartilage: structure, function, and importance in tissue engineering. Crit Rev Biomed Eng 35: 363-411.

Rieppo L, Rieppo J, Jurvelin JS, Saarakkala S (2012) Fourier transform infrared spectroscopic imaging and multivariate regression for prediction of proteoglycan content of articular cartilage. PLoS One 7: DOI: 10.1371/journal.pone.0032344.

Saarakkala S, Wang SZ, Huang YP, Zheng YP (2009) Quantification of the optical surface reflection and surface roughness of articular cartilage using optical coherence tomography. Phys Med Biol 54: 6837-6852.

Schindelin J, Rueden CT, Hiner MC, Eliceiri KW (2015) The ImageJ ecosystem: an open platform for biomedical image analysis. Mol Reprod Dev 82: 518529.

Setton LA, Mow VC, Muller FJ, Pita JC, Howell DS (1993) Altered structure-function relationships for articular cartilage in human osteoarthritis and an experimental canine model. Agents Actions Suppl 39: 27-48.

Sherlock B, Yu F, Stone J, Warren S, Paterson C, Neil MA, French PM, Knight J, Dunsby C (2016) Tunable fibre-coupled multiphoton microscopy with a negative curvature fibre. J Biophotonics 9: 715-720.

Squires GR, Okouneff S, Ionescu M, Poole AR (2003) The pathobiology of focal lesion development in aging human articular cartilage and molecular matrix changes characteristic of osteoarthritis. Arthritis Rheum 48: 1261-1270.

Sun Y, Park J, Stephens DN, Jo JA, Sun L, Cannata JM, Saroufeem RM, Shung KK, Marcu L (2009) Development of a dual-modal tissue diagnostic system combining time-resolved fluorescence spectroscopy and ultrasonic backscatter microscopy. Rev Sci Instrum 80: 065104.

Sun Y, Responte D, Xie H, Liu J, Fatakdawala H, $\mathrm{Hu}$ J, Athanasiou KA, Marcu L (2012) Nondestructive evaluation of tissue engineered articular cartilage using time-resolved fluorescence spectroscopy and ultrasound backscatter microscopy. Tissue Eng Part C Methods 18: 215-226.

Wagnieres GA, Star WM, Wilson BC (1998) In vivo fluorescence spectroscopy and imaging for oncological applications. Photochem Photobiol 68: 603-632.

Woessner JF Jr. (1961) The determination of hydroxyproline in tissue and protein samples containing small proportions of this imino acid. Arch Biochem Biophys 93: 440-447.

Yankelevich DR, Ma D, Liu J, Sun Y, Sun Y, Bec J, Elson DS, Marcu L (2014) Design and evaluation of a device for fast multispectral time-resolved fluorescence spectroscopy and imaging. Rev Sci Instrum 85: DOI: 10.1063/1.4869037.

Zhang L, Hu J, Athanasiou KA (2009) The role of tissue engineering in articular cartilage repair and regeneration. Crit Rev Biomed Eng 37: 1-57.

\section{Discussion with Reviewer}

Stephen Waldman: Can the authors comment on the effect of the water content in the tissue samples (as TRFS was highly correlated with GAG content relative to tissue wet weight as opposed to FLIm, which was highly correlated with collagen content relative to tissue dry weight)?

Authors: In this study, all optical measurements were taken on hydrated tissue. The normalization of ECM proteins to wet or dry weight was chosen based on standard reporting conventions and Table 1 allows conversion between the two methods. Because water does not have a detectable fluorescent signal, the water itself does not affect fluorescence the lifetime values used in our analysis.

Stephen Waldman: If the water content of the tissue is not determined, does this pose any limitation to the technique?

Authors: No, we do not see any limitation.

Editor's note: The Scientific Editor responsible for this paper was Stephen Ferguson. 